\title{
Severe neurological manifestations of influenza during 2018-2019 influenza season: Case series of 13 pediatric patients
}

\author{
M. Gultekin Kutluk M.D. ${ }^{a}$ and E. Naz Kadem M.D. ${ }^{b}$
}

\begin{abstract}
Influenza is mostly associated with the respiratory tract system, especially in the winter season. Various neurological complications could occur due to influenza infection. Pediatric patients who had severe neurological manifestations due to influenza infection from September 2018 to February 2019 were evaluated for clinical characteristics, neuroimaging studies, treatment, and outcome. We aimed to assess Influenzaassociated encephalitis in children, emphasize different neurological manifestations and neuroimaging changes. Thirteen patients were included in the study. Neurological symptoms occurred after flu-like symptoms. Neuroimaging changes of influenza-associated encephalitis/encephalopathy include cortical and subcortical white matter signal alterations, localized or generalized edema, and bilateral symmetrical multifocal lesions on the thalamus and cerebellar medulla. Pulse methylprednisolone, intravenous immunoglobulin, plasma exchange, and oseltamivir are the therapy choices. It is essential to consider influenza-associated encephalitis in patients with seizures, encephalopathy with supporting radiological findings, especially during the influenza season and starting treatment as fast as possible for better outcomes. Keywords:encephalitis, encephalopathy, Guillain-Barrasyndrome, corpus callosum, pediatrics.
\end{abstract}

http:/ / dx.doi.org/10.5546/ aap.2021.eng.e142

To cite: Kutluk MG, Kadem EN. Severe neurological manifestations of influenza during 2018-2019 influenza season: Case series of 13 pediatric patient. Arch Argent Pediatr 2021;119(2):e142-e148.

a. Pediatric Neurology Department.

b. Pediatrics Clinic.

Antalya Research and Training Hospital, Antalya, Turkey.

E-mail address:

M. Gultekin Kutluk M.D: gultekinkutluk@gmail.com

Funding: None.

Conflict of interest: None.

Received: 5-20-2020

Accepted: 10-23-2020

\author{
GLOSSARY \\ ADC: Apparent diffusion coefficient. \\ CSF: Cerebrospinal fluid. \\ EEG: Electroencephalography. \\ FLAIR: Fluid-attenuated inversion recovery. \\ GOSE: Glasgow outcome scale expanded. \\ IAE: Influenza-associated encephalitis. \\ IVIG: Intravenous immunoglobulin. \\ MP: Methylprednisolone. \\ MRI: Magnetic resonance imaging. \\ RIDT: Rapid influenza diagnostic tests.
}

\section{INTRODUCTION}

Children have influenza infection mostly with respiratory tract system symptoms, and are hospitalized due to respiratory and neurological complications, especially in the winter. Almost 90 million children are infected with influenza every year worldwide. ${ }^{1,2}$ Incidence of influenzaassociated neurological complications show difference upon regions; Glaser et al., reported neurological complications of 1.2/1000 000 . In Japan, the incidence of influenza-associated encephalitis (IAE) was reported as 6-11/1 000000 in 1999-2000. ${ }^{3}$ Neurological manifestations vary in a broad spectrum of severity, and are characterized by specific magnetic resonance imaging (MRI) findings. There are also many cases with different MRI changes that are not common in the literature. ${ }^{2}$ Here in this study, we presented 13 pediatric cases with IAE during the 2018-2019 influenza season in a single center. This study marks on different severity and neuroimaging characteristics of IAE and treatment options.

\section{METHODS}

\section{Study population}

Patients aged 6 months to 18 years, admitted to a single center for influenza A infections with a severe neurological complication from September 2018 to December 2019, were retrospectively investigated. Patients' written consents were obtained from their legal guardians by the corresponding author. 


\section{Virology survey}

Real-time reverse transcription-polymerase chain reaction (RT-PCR) and rapid influenza diagnostic tests (RIDT) were performed with the airway and cerebrospinal fluid (CSF) specimens.

\section{Neuroimaging and electrophysiological studies}

Electroencephalography (EEG) was performed for all patients and evaluated by the same pediatric neurologist. MRI with T1 and T2 weighted images, fluid-attenuated inversion recovery (FLAIR), diffusion-weighted imaging (DWI), and apparent diffusion coefficient (ADC, $\mathrm{b}=1000$ ) studies were acquired by $1.5 \mathrm{~T}$ or $3 \mathrm{~T} \mathrm{MR}$ machines. Gadolinium contrast medium was used for the demonstration of inflammation.

\section{Clinical evaluation}

All patients were evaluated and treated by pediatric neurology, pediatric infectious diseases, and pediatric intensive care unit (PICU) departments. Glasgow coma scale (GCS) was used to describe the general level of consciousness. ${ }^{4}$ Glasgow Outcome Scale Expanded (GOSE) was used to determine the outcome at discharge from the hospital. ${ }^{5}$

\section{RESULTS}

\section{Demographic data}

Thirteen patients were included in the study. Demographic data for all patients are shown in Table 1. Prior to the presentation, patients 5, 9, and 11 had medical comorbidities, and the rest

TABLE 1. Demographic data, first presentation characteristics and clinical course, outcome of IAE patients

\begin{tabular}{|c|c|c|c|c|c|c|c|c|c|}
\hline $\begin{array}{l}\text { Case } \\
\text { Number }\end{array}$ & Age/Sex & $\begin{array}{c}\text { Respiratory } \\
\text { track symptom } \\
\text { onset (days) }\end{array}$ & $\begin{array}{l}\text { Clinical } \\
\text { Presentation }\end{array}$ & $\begin{array}{c}\text { GCS at } \\
\text { first } \\
\text { presentation }\end{array}$ & Vaccine & EEG & $\begin{array}{l}\text { Days } \\
\text { in PICU }\end{array}$ & $\begin{array}{c}\text { GOSE } \\
\text { (after } \\
\text { treatment) }\end{array}$ & $\begin{array}{l}\text { Neurological } \\
\text { sequela }\end{array}$ \\
\hline 1 & $21 \mathrm{~m} / \mathrm{F}$ & 7 & $\begin{array}{l}\text { Fever, coryza, seizures, } \\
\text { altered mental status }\end{array}$ & 10 & No vaccine & Encephalopathic & 8 & 8 & None \\
\hline 2 & $11 \mathrm{y} / \mathrm{F}$ & 15 & $\begin{array}{l}\text { Dysphagia, aphasia, } \\
\text { altered mental status }\end{array}$ & 12 & No Vaccine & Encephalopathic & 16 & 7 & None \\
\hline 3 & $8 \mathrm{y} / \mathrm{M}$ & 10 & $\begin{array}{l}\text { Dysphagia, aphasia, } \\
\text { altered mental status }\end{array}$ & 13 & No vaccine & Encephalopathic & 17 & 8 & None \\
\hline 4 & $7 \mathrm{y} / \mathrm{F}$ & 4 & Transient visual loss & 15 & No vaccine & Not performed & 0 & 8 & None \\
\hline 5 & $16 \mathrm{y} / \mathrm{M}$ & 6 & Seizures, altered mental status & 11 & No vaccine & Encephalopathic & 23 & 6 & $\begin{array}{l}\text { Epilepsy } \\
\text { and } \\
\text { behavioral } \\
\text { disorder }\end{array}$ \\
\hline 6 & $8 \mathrm{y} / \mathrm{M}$ & 4 & Seizures, altered mental status & 12 & No vaccine & Encephalopathic & 4 & 8 & None \\
\hline 7 & $12 \mathrm{y} / \mathrm{F}$ & 5 & $\begin{array}{l}\text { Headache, aphasia, seizures, } \\
\text { altered mental status }\end{array}$ & 13 & No vaccine & Encephalopathic & 11 & 8 & None \\
\hline 8 & $10 \mathrm{y} / \mathrm{M}$ & 4 & Seizures, altered mental status & 12 & No vaccine & Encephalopathic & 3 & 8 & None \\
\hline 9 & $18 \mathrm{~m} / \mathrm{M}$ & 2 & $\begin{array}{l}\text { Fever, coryza, seizures, } \\
\text { altered mental status }\end{array}$ & 6 & No vaccine & Convulsive status & 5 & 8 & None \\
\hline 10 & $6 y / M$ & 15 & $\begin{array}{l}\text { Dystonia, aphasia, } \\
\text { loss of consciousness }\end{array}$ & 9 & No vaccine & Encephalopathic & 20 & 6 & $\begin{array}{c}\text { Intellectual } \\
\text { disability, } \\
\text { behavioral } \\
\text { disorder, } \\
\text { walking } \\
\text { difficulties }\end{array}$ \\
\hline 11 & $16 y / F$ & 11 & $\begin{array}{l}\text { Dystonia, aphasia, } \\
\text { loss of consciousness }\end{array}$ & 8 & No vaccine & $\begin{array}{l}\text { Encephalopathic, } \\
\text { centrotemporal } \\
\text { epileptic activity }\end{array}$ & 44 & 5 & $\begin{array}{c}\text { Intellectual } \\
\text { disability } \\
\text { spastic } \\
\text { tetraparesis }\end{array}$ \\
\hline 12 & $9 \mathrm{y} / \mathrm{M}$ & 7 & Delirium, altered mental status & 9 & No vaccine & Encephalopathic & 29 & 8 & None \\
\hline 13 & $8 y / F$ & 15 & Ataxia & 15 & No vaccine & Not performed & 0 & 8 & None \\
\hline
\end{tabular}

GCS: Glasgow coma score; EEG: electroencephalography; PICU: pediatric intensive care unit;

GOSE: Glasgow outcome scale expanded; F: female; M: male. 
were healthy. Patient 5 had epilepsy, and patient 11 had mental retardation. Patient 9 was born prematurely. Three patients had consanguineous parents.

\section{Neurological symptoms}

All patients developed neurological symptoms after a mean of 8.07 days (min. 4 days - max. 15 days) of flu-like symptoms. The first presentation was the altered state of consciousness in 11 of 13 patients, and GCS was mean 11.15 (min. 6 - max. 15). Four of them gained consciousness after day 1 , while 7 of them had persistent high cortical function impairment. One patient developed urine retention, which was considered as autonomic dysfunction (P2). Six were presented with seizures, and patient 9 had status epilepticus (Table 1).

\section{Laboratory findings}

Influenza A was detected by PCR (10 patients, $76.9 \%$ ) or RIDT (3 patients, $23.1 \%$ ) from the nasal swab. No microorganisms were detected from neither blood nor CSF samples. There was no other abnormality in laboratory studies related to the mentioned clinical features. (Table 2)

\section{Neuroimaging examinations and EEG findings}

Pathological EEG studies were reported as encephalopathy, centrotemporal epileptic activity, and convulsive status (Table 1). Patient 5 had frontotemporal epileptic activity on the follow-up, and patient 11 had centrotemporal epileptic activity on EEG. Cranial MRI scans of patient 4 showed signal alterations in the splenium of the corpus callosum, which was hyperintense on T2WI-FLAIR and restricted diffusion, isointense on T1WI with no contrast enhancement (Figure 1A-D1). Consequently, she was diagnosed with mild encephalitis/ encephalopathy with a reversible splenial lesion of corpus callosum (MERS). MRI changes were alike in three cases (Patients 2, 3 and 7). All showed bilaterally swollen nucleus caudatus, and lentiform nuclei with T2/ FLAIR hyperintensity (Figure 1A-A1, B1, C1), and this lesion demonstrated restricted diffusion with decreased signal intensity in ADC maps. Patient 10 had signal alterations in the pons, mesencephalon, medulla oblongata, and cerebellar peduncles with T2 / FLAIR hyperintensity and restricted diffusion with decreased signal intensity in ADC maps of the pons (Figure 1A-E1). MRI scans of patient 11 showed hemorrhagic changes at the bilateral thalamus, mesencephalon, and cerebellar hemispheres. T2 / FLAIR images showed hyperintensity at bilateral thalamus, basal ganglia, mesencephalon, pons and, periventricular white matter (Figure 1A-F1). Diffusion-weighted ADC map images showed

TABLE 2. Laboratory findings

\begin{tabular}{lcccc}
\hline Case number & CSF & Influenza type & Blood culture & Autoimmune encephalitis panel \\
\hline 1 & Normal & Influenza A (PCR) & Sterile & - \\
2 & Normal & Influenza A (H1N1) (PCR) & Sterile & Normal \\
3 & Pleocytosis & Influenza A (H1N1) (PCR) & Sterile & - \\
4 & Normal & Influenza A (H1N1) (RIDT) & Sterile & - \\
5 & Normal & Influenza A (PCR) & Sterile & - \\
6 & Normal & Influenza A (PCR) & Sterile & - \\
7 & Normal & Influenza A (PCR) & Sterile & - \\
8 & Normal & Influenza A (RIDT) & Sterile & - \\
9 & Normal & Influenza A (RIDT) & Sterile & Normal \\
10 & Normal & Influenza A (PCR) & Sterile & Normal \\
11 & Normal & Influenza A (PCR) & Sterile & Normal \\
12 & Normal & Influenza A (PCR) & Sterile & - \\
13 & Albumin-cytologic & Influenza A (PCR) & Sterile & \\
& dissociation & & & \\
\hline
\end{tabular}

Autoimmune encephalitis panel: N-methyl-D-aspartate receptor, anti-glutamate type $\alpha$-amino-3-hydroxy-5-methyl-4isoxazolepropionic acid 1 and 2, anti-contactin-associated protein-like 2, anti-gamma-aminobutyric acid B receptor, anti-leucine-rich, glioma inactivated 1 antibody.

CSF: cerebrospinal fluid; PCR: polymerase chain reaction; RIDT: rapid influenza diagnostic tests. 
restricted diffusion in the bilateral thalamus, posterior horn of internal capsule, and frontoparietal periventricular white matter. MRI studies of patient 5 demonstrated giral expansion and edema in the left frontotemporoparietal area on pre-contrast studies with T2/FLAIR hyperintensity. Also, there was a leptomeningeal enhancement on post-contrast FLAIR images. Patient 13 showed contrast enhancement on the conus medullaris and the nerve roots of the cauda equina. Follow-up MRIs showed improvement (Figure 1B).

\section{Treatment}

Acyclovir and oseltamivir were given as antiviral treatment until microbiological results came back. Eleven patients received antiviral treatment within the first 12 hours. Patient 5 was transferred to our hospital and given antiviral treatment after 72 hours of the first presentation to medical attention. Pulse methylprednisolone (MP) was given to eleven patients with an initiation time of $18,5 \mathrm{~h}$. Intravenous immunoglobulin (IVIG) was applied to seven cases, which were more advanced and had more severe MRI changes. Also, the patient diagnosed with Guillain-Barré syndrome received IVIG. Plasma exchange was also performed for six patients who partially responded to pulse MP and IVIG treatments. Hypertonic saline infusion was administered as anti-edema treatment. Anticonvulsant therapy was given to six patients who had seizures, and two patients for prevention (Table 3).

\section{Management and outcome}

Eleven patients required PICU stay for a mean of 16.3 days (min. 3 days-max. 44). All patients were discharged from the hospital with a mean 7.3 GOSE (min. 5-max. 8) and called for monthly follow-ups in 9 months. Patients 5 and 11 developed epilepsy and required anticonvulsant treatment (levetiracetam and valproic acid). Also, patients 10 and 11 showed behavioral changes, regression of cognitive functions, and walking difficulties (Table 1). Six patients had physiotherapy after discharge.

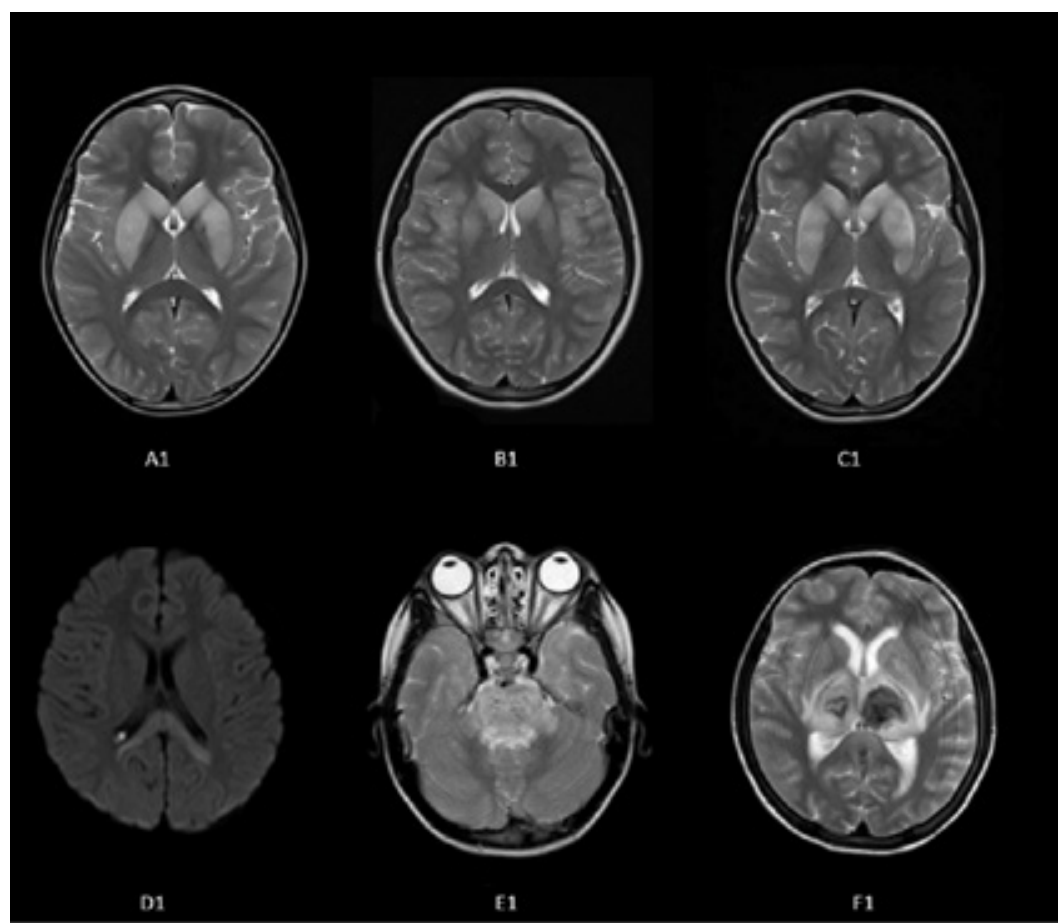

Bilaterally swollen nucleus caudatus and lentiform nuclei with T2/FLAIR hyperintensity (A1, B1, C1), signal alterations in the splenium of corpus callosum, which was hyperintense on T2WI-FLAIR (D1). Pons and cerebellar peduncle with T2/FLAIR hyperintensity (E1), bilateral thalamus with T2/FLAIR hyperintensity and hemorrhagic changes (F1). Patients 1, 6, 8, 9, and 12 had normal MRI findings. 


\section{DISCUSSION}

Influenza is one of the most common causes of pediatric acute encephalitis / encephalopathy. Influenza-associated encephalitis / encephalopathy could be seen in all ages, from infancy to puberty. ${ }^{4}$
Influenza epidemics show a characteristic seasonal pattern with peak incidence occurring in fall and winter. ${ }^{6}$ Live attenuated and inactivated vaccines reduce the risk of influenza infection in children. ${ }^{7}$ However, there is no certain evidence between influenza vaccination and protection

TABLE 3. Treatment of IAE patients

\begin{tabular}{lcccccc}
\hline Case number & Oseltamivir & Acyclovir & Pulse MP & IVIG $(\mathbf{2}$ g/kg) & Plasmapheresis & Antiepileptic \\
\hline 1 & + & + & $+/ 5$ days & - & - & Levetiracetam \\
2 & + & + & $+/ 5$ days & $+/ 4$ days & $+/ 7$ days & - \\
3 & + & $+/ 5$ days & $+/ 4$ days & $+/ 7$ days & - \\
4 & + & $+/ 5$ days & - & - & - \\
5 & + & + & $+/ 4$ days & - & Levetiracetam $/$ \\
& + & + & $+/ 5$ days & - & - & Valproic acid \\
6 & + & $+/ 5$ days & $+/ 4$ days & $+/ 7$ days & Levetiracetam \\
7 & + & $+/ 5$ days & - & - & Phenytoin \\
8 & + & + & - & - & - & Levetiracetam \\
9 & + & + & $+/ 5$ days & $+/ 4$ days & $+/ 7$ days & Levetiracetam \\
10 & + & + & $+/ 5$ days & $+/ 4$ days & $+/ 7$ days & Levetiracetam \\
11 & + & + & $+/ 5$ days & $+/ 4$ days & $+/ 7$ days & Levetiracetam \\
12 & - & - & $+/ 4$ days & - & - \\
13 & + & + & & & -
\end{tabular}

MP: methylprednisolone; IVIG: intravenous immunoglobulin.

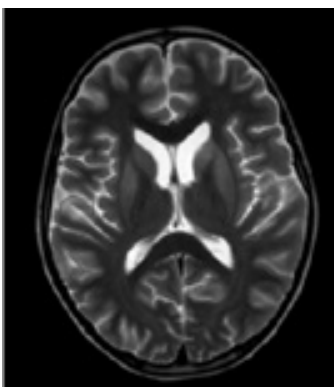

A2

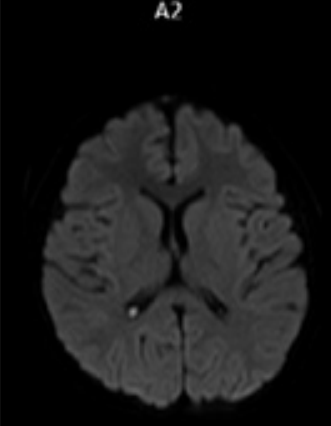

D2

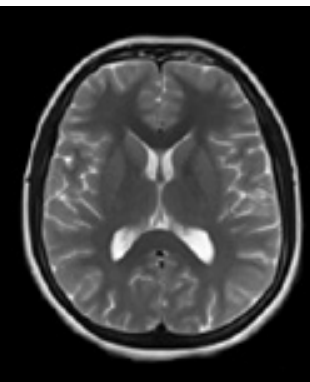

B?

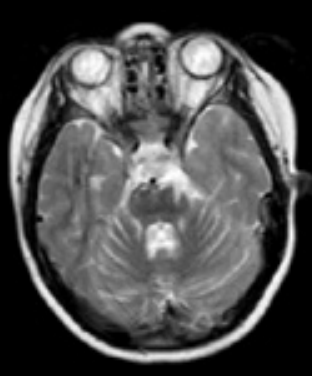

E2

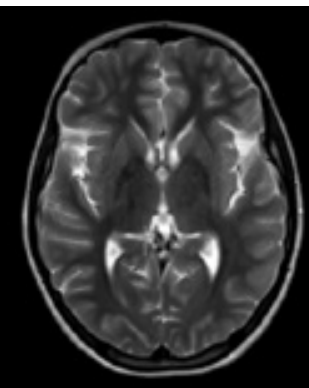

c?

After treatment, improved (A2-E2). Patient 11's follow-up MRI study wasn't performed because of COVID-19 pandemic. 
against neurological complications. ${ }^{3}$

The underlying mechanism of influenza encephalitis is controversial. There are different opinions on pathogenesis; viral invasion, certain genetic backgrounds and cytokine storm could influence the CNS function, and damage the CNS tissue. ${ }^{8}$ Fujimoto et al. reported that influenza virus RNA was detected in the CSF, ${ }^{9}$ contrary, in other reports, only a few patients were positive for viral RNA in CSF. ${ }^{8}$ Viral RNA was only detected in nasal swab samples of our patients. With our evidence, we managed our patients' treatment as an immune-related disease, not as the primary infection.

Neuroimaging changes of influenza-associated encephalitis / encephalopathy include cortical and subcortical white matter signal alterations, localized or generalized edema, and bilateral symmetrical multifocal lesions on the thalamus and cerebellar medulla. ${ }^{10}$ Different clinicalneuroimaging diagnoses are the following; ANE (acute necrotizing encephalopathy), PRES (posterior reversible encephalopathy syndrome), ADEM (acute disseminated encephalomyelitis), MERS, meningoencephalitis, AESD (acute encephalopathy with biphasic seizures and late diffusion restriction), HSES (hemorrhagic shock and encephalopathy syndrome). ${ }^{11,12}$ MRI studies of patients 4, 5, 10, 11 and 13 showed similarity to literature. Patients 2, 3 and 7 showed bilateral nucleus caudatus and putamen involvements in MRI. Isolated bilateral nucleus caudatus and putamen involvements are not frequently seen in published literature. ${ }^{2,4,12,13}$

There is no specific treatment for IAE, and there are several aspects of treatment, including antiviral therapy, high dose corticosteroids, IVIg, and plasma exchange. ${ }^{1}$ The efficiency of oseltamivir on favorable outcomes is not absolute, but Infectious Disease Society of America guidelines recommend oseltamivir, and some series have supporting evidence. ${ }^{5,14}$ Pulse MP, IVIG, and plasma exchange are the first line immunotherapy for autoimmune encephalitis. ${ }^{15}$ Immune-related mechanisms are considered as the underlying pathogenesis for IAE; therefore, pulse MP and IVIG are the frequently used treatment options. ${ }^{4}$

Among surviving patients of IAE, neurological deficits were reported in 21-45\%.1,3 Specific prognostic factors are not determined for $\mathrm{IAE}^{3}$ early diagnosis was the most relevant factor in our cases. MRI is essential to make an early diagnosis and treatment. Patient 11 had the most severe MRI findings and life-threatening clinical features. She was discharged with a GOSE score of 5 , and on the follow-up, she was able to walk with support. Patient 5 received treatment later than others, his GOSE score was low (6), and he developed neurological sequelae. GOSE scores were high (GOSE 7,8) for the rest and sequelaefree on follow-ups. We think early recognition, early and intense therapy are important on morbidity and mortality with this experience.

In this paper, severe neurological complications of influenza were presented as different MRI findings and associated clinical features. Recognition of neurological complications of influenza is difficult, especially when the presentation is not typical. MRI studies are highly important for early diagnosis and treatment. Nucleus caudatus and putamen involvements on MRI are unusual for IAE. Nevertheless, during the influenza season, these involvements should be considered carefully with clinical and laboratory evaluations and start treatment as early as possible.

\section{Acknowledgment}

We are grateful for all the hard work of pediatric infectious diseases and pediatric intensive care unit departments.

\section{REFERENCES}

1. Paksu MS, Aslan K, Kendirli T, Akyildiz BN, et al. Neuroinfluenza: evaluation of seasonal influenza associated severe neurological complications in children (a multicenter study). Childs Nerv Syst. 2018; 34(2):335-47.

2. Dadak M, PulR, Lanfermann H, Hartmann H, et al. Varying Patterns of CNS Imaging in Influenza A Encephalopathy in Childhood. Clin Neuroradiol. 2019; 30(2):243-9.

3. Britton PN, Blyth CC, Macartney K, Dale RC, et al. The Spectrum and Burden of Influenza-Associated Neurological Disease in Children: Combined Encephalitis and Influenza Sentinel Site Surveillance From Australia, 2013-2015. Clin Infect Dis. 2017; 65(4):653-60.

4. Chen LW, Teng CK, Tsai YS, Wang JN, et al. Influenzaassociated neurological complications during 2014-2017 in Taiwan. Brain Dev. 2018; 40(9):799-806.

5. Goenka A, MichaelBD, Ledger E, Hart IJ, et al. Neurological manifestations of influenza infection in children and adults: results of a National British Surveillance Study. Clin Infect Dis. 2014; 58(6):775-84.

6. Gómez-BarrosoD, León-GómezI, Delgado-Sanz C, Larrauri A. Climatic Factors and Influenza Transmission, Spain, 2010-2015. Int J Environ Res Public Health. 2017; 14(12):1469.

7. Jefferson T, Rivetti A, Di Pietrantonj C, Demicheli V. Vaccines for preventing influenza in healthy children. Cochrane Database Syst Rev. 2018; 2(2):CD004879.

8. Wang GF, Li W, Li K. Acute encephalopathy and encephalitis caused by influenza virus infection. Curr Opin Neurol. 2010; 23(3):305-11.

9. Fujimoto S, Kobayashi M, Uemura O, Iwasa M, et al. PCR on cerebrospinal fluid to show influenza-associated acute 
encephalopathy or encephalitis. Lancet. 1998;352(9131):873-5.

10. Surana P, TangS, McDougall M, Tong CY, etal. Neurological complications of pandemic influenza A H1N1 2009 infection: European case series and review. Eur J Pediatr. 2011; 170(8):1007-15.

11. Wilking AN, Elliott E, García MN, Murray KO, Munoz FM. Central nervous system manifestations in pediatric patients with influenza A H1N1 infection during the 2009 pandemic. Pediatr Neurol. 2014; 51(3):370-6.

12. Amin R, Ford-Jones E, Richardson SE, MacGregor D, et al. Acute childhood encephalitis and encephalopathy associated with influenza: a prospective 11-year review.
Pediatr Infect Dis J. 2008; 27(5):390-5.

13. Britton PN, Dale RC, Blyth CC, Macartney K, et al. Influenzaassociated Encephalitis / Encephalopathy Identified by the Australian Childhood Encephalitis Study 2013-2015. Pediatr Infect Dis J. 2017; 36(11):1021-6.

14. Tunkel AR, Glaser CA, Bloch KC, Sejvar JJ, et al. The management of encephalitis: clinical practice guidelines by the Infectious Diseases Society of America. Clin Infect Dis. 2008; 47(3):303-27.

15. Shin YW, Lee ST, Park KI, Jung KH, et al. Treatment strategies for autoimmune encephalitis. Ther Adv Neurol Disord. 2018; 11:1756285617722347. 